\title{
ON GALOIS EXTENSION OF RINGS
}

\author{
TERUO KANZAKI
}

To the memory of TADASI NAKayama

1. Introduction. Let $A$ be a ring and $G$ a finite group of ring automorphisms of $A$. The totality of elements of $A$ which are left invariant by $G$ is a subring of $A$. We call it the $G$-fixed subring of $\Lambda$. Let $\Delta=\Delta(\Lambda, G)=\sum_{\sigma \in G} \oplus A u$, be the crossed product of $A$ and $G$ with trivial factor set, i.e. $\left\{\boldsymbol{u}_{\sigma}\right\}$ is a $A$-free basis of $\Delta$ and $\boldsymbol{u}_{\sigma} \boldsymbol{u}_{\tau}=\boldsymbol{u}_{\tau \tau}, \boldsymbol{u}_{\tau} \lambda=\sigma(\lambda) \boldsymbol{u}_{3}$ for $\lambda \in A$, and let $\Gamma$ be a subring of the $G$-fixed subring of $A$ which has the same identity as $A$. Then we have a ring homomorphism

$$
\delta: \Delta(\Lambda, G) \rightarrow \operatorname{Hom}_{\Gamma}^{r}(\Lambda, \Lambda)
$$

defined by $\delta\left(\lambda u_{\sigma}\right)(x)=\lambda \sigma(x)$, where $\operatorname{Hom}_{\Gamma}^{r}(\Lambda, \Lambda)$ is the $\Gamma$-endomorphism ring of $\Lambda$ regarded as $\Gamma$-right module.

In [4], we generalized the notion of Galois extension, which was first defined by Auslander and Goldman [1] for commutative rings, to non commutative case, and discussed the Galois theory for non commutative rings. Our definition of Galois extension is as follows. A ring $A$ is called a Galois extension of $\Gamma$ relative to $G$ if the following conditions are satisfied:

I. $\Gamma$ is the $G$-fixed subring of $\Lambda$,

Il. $\Lambda$ is a finitely generated projective $\Gamma$-right module,

III. $\delta$ is an isomorphism of $\left.\Delta^{\prime} \Lambda, G\right)$ to $\operatorname{Hom}_{\Gamma}^{r}(\Lambda, \Lambda)$.

On the other hand, Chase, Harrison and Rosenberg [3] gave another definition of Galois extension, which is equivalent to the above in commutative case, and developed a Galois theory for commutative rings. In order to state other definition, we set $\operatorname{Tr}(x)=\sum_{\sigma \in G} \sigma(x)$ for $x \in \Lambda$. Then $\Lambda$ is called to be a Galois extension of $\Gamma$ relative to $G$ if the following two conditions are satisfied:

CHR I. $\Gamma=\operatorname{Tr}(\Lambda)$.

CHR II. There exist $x_{1}, x_{2}, \ldots$ and $x_{r}$ and $y_{1}, y_{2}, \ldots y_{r}$ in $A$ such that

Received February 8, 1965. 
for $\sigma \in G \sum_{i} x_{i} \sigma\left(y_{i}\right)=\left\{\begin{array}{l}\mathrm{I}, \text { if } \sigma=1 \\ 0, \text { if } \sigma \neq 1 .\end{array}\right.$

In $\S 2$, we discuss the relationship between these two definitions of Galois extension and we shall show that if $\Lambda$ and $\Gamma$ are algebras over a commutative ring $R$ and $\Gamma$ is $R$-separable then they are equivalent to each other. In $\S 3$, we shall give an improvement of the Galois theory established in [4] which is also a generalization of the Galois theory in [3] to non commutative case. In $\S 4$, for a Galois extension $\Lambda$ of $\Gamma$ relative to $G$, we consider a ring-automorphism $\rho$ of $\Lambda$ which leaves invariant each element of $\Gamma$ and we shall show that $\rho$ is an element of $G$ under some assumption.

2. Galois extension. Throughout this section, $\Lambda$ stands for a ring with identity, $G$ a finite group of ring automorphisms of $A$ and $\Gamma$ a subring of $\Lambda$ which has the same identity as $\Lambda$. We shall call $\Lambda$ a Galois extension of $\Gamma$ relative to $G$ if the three condition I, II, III in $\S 1$ are satisfied. $A$ is regarded as $\Delta(A, G)$ - left module through $\delta$. Then a right multiplication of an element $\gamma$ of $\Gamma$ induces a $\Delta(\Lambda, G)$ - endomorphism of $\Lambda$ if $\Gamma$ is a subring of the $G$-fixed subring of $\Lambda$ We shall denote it by $\gamma_{r}$ and set $\left\{\gamma_{r} \mid \gamma \in \Gamma\right\}=\Gamma_{r}$. Then the condition $I$ is equivalent to

$I^{\prime}$.

$$
\Gamma_{r}=\operatorname{Hom}_{\Delta}^{l}(\Lambda, \Lambda) .
$$

The following lemma is proved in [4].

Lemma 2.1. $A$ is a Galois extension of $\Gamma$ relative to $G$ if and only if $\Gamma_{r}=$ $\operatorname{Hom}_{\Delta}^{l}(\Lambda, \Lambda)$ and $\Delta(\Lambda, G)=\Lambda u \Lambda$, where $u=\sum_{\sigma \in G} u_{\sigma}$.

Lemma 2.2. $\Lambda u \Lambda=\Delta(\Lambda, G)$ if and only if the condition CHR II holds.

Proof. Since $\Lambda u \Lambda$ is a two sided ideal of $\Delta(\Lambda, G), \Delta(\Lambda, G)=\Lambda u \Lambda$ if and only if $1 \in \Lambda u \Lambda$. But $1 \in \Lambda u \Lambda$ if and only if there exist $x_{1}, x_{2}, \ldots x_{r}, y_{1}, y_{2}, \ldots y_{r}$ in $\Lambda$ such that $1=\sum_{i=1}^{r} x_{i} u y_{i}=\sum_{i=1, \sigma \in G}^{r} x_{i} \sigma\left(y_{i}\right) u_{\sigma}$. Thus we obtain this lemma.

Proposition 2.3. $\operatorname{Tr}(\Lambda)=\operatorname{Hom}_{\Delta}^{l}(\Lambda, \Lambda)$ if and only if $\Lambda$ is a finitely generated projective $\Delta$-module.

Proof. In Lemma 3 in [4], we obtained the isomorphism $\kappa ; \operatorname{Hom}_{\Delta}^{7}(A, \Delta) \rightarrow$ $\boldsymbol{u} \Lambda$, defined by $\kappa(f)=f(1)$. For the homomorphism $r: \operatorname{Hom}_{\Delta}^{l}(\Lambda, \Delta) \rightarrow \operatorname{Hom}_{\Delta}^{l}(\Lambda, \Lambda)$ defined by $r(f)(\lambda)=f(\lambda) 1$, the following diagram is commutative; 


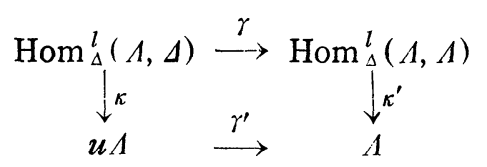

where $\kappa^{\prime}$ is a monomorphism defihed by $\kappa^{\prime}(f)=f(1)$ for $f \in \operatorname{Hom}_{\Delta}^{l}(\Lambda, \Lambda)$, and $\gamma^{\prime}$ is a homomorhpism defined by $\gamma^{\prime}(u \lambda)=(u \lambda) 1=\operatorname{Tr}(\lambda)$ for $u \lambda \in u A$. Since $\operatorname{Im}\left(\gamma^{\prime} \kappa\right)=\operatorname{Im}\left(\gamma^{\prime}\right)=\operatorname{Tr}(\Lambda), \operatorname{Im}\left(\kappa^{\prime}\right)=\operatorname{Tr}(\Lambda)$ if and only if $\gamma$ is an epimorphism. On the other hand, for epimorphism $\tau: \operatorname{Hom}_{\Delta}^{l}(\Lambda, \Lambda) \rightarrow \operatorname{Hom}_{\Delta}^{l}(\Lambda, \Delta) \otimes{ }_{\Delta} \Lambda$ defined by $\tau(f)=f \otimes 1$, and for the homomorphism $\mu: \operatorname{Hom}_{\Delta}^{l}(\Lambda, \Delta) \otimes \Delta \Lambda \rightarrow \operatorname{Hom}_{\Delta}^{l}(\Lambda, \Delta)$ defined by $\mu(f \otimes \lambda)(x)=f(x) \lambda$, we have the following commutative diagram:

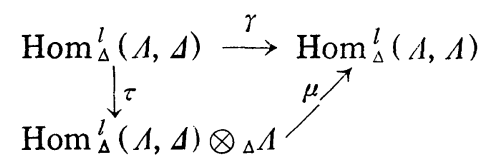

Because, for $f \in \operatorname{Hom}_{\Delta}^{l}(\Lambda, \Lambda), \mu \tau(f)(\lambda)=\mu(f \otimes 1)(\lambda)=f(\lambda) 1=\gamma(f)(\lambda)$. Therefore $\gamma$ is an epimorphism if and only if $\mu$ is an epimorphism. But by Proposition A. 1 in [2], $\mu$ is an epimorphism if and only if $\Lambda$ is a finitely generated projective $\Delta$-module. Therefore we obtain this proposition.

Proposition 2.4. a) $\Lambda$ is a Galois extension of $\Gamma$ relative to $G$ if and only if the condition I and CHR II. are satisfied. b) The condition CHR I. holds if and only if the condition I holds and $\Lambda$ is a finitely generated projective 4-module.

Proof. a) is obtained in above. b) Since $\operatorname{Tr}(\Lambda)$ is a two sided ideal of the $G$-fixed subring of $\Lambda . \operatorname{Tr}(\Lambda)=\Gamma$ if and only if $\operatorname{Tr}(\Lambda)_{r}=\operatorname{Hom}_{\Delta}^{l}(\Lambda, \Lambda)$ and $\Gamma_{r}=$ $\operatorname{Hom}_{\Delta}^{l}(\Lambda, \Lambda)$. Therefore b) follows from Proposition 2.3.

THEOREM 2.5. Let $\Gamma \subset A$ be algebras over a commutative ring $R$, and let $\Gamma$ be separable over $R$. Then $A$ is a Galois extension of $\Gamma$ relative to $G$ if and only if the conditions CHR I. and CHR II. hold.

Proof. If $\Gamma$ is separable over $R$ and $\Lambda$ is a Galois extension of $I$ relative to $G$, then $\Delta=\operatorname{Hom}_{\Gamma}^{r}(\Lambda, A)$ is separable over $R$ and $\Lambda$ is a finitely generated projective $\Delta$-module by Colrollary 1 in [4]. Therefore by Proposition 2.4 CHR I. and CHR II. hold. The converse follows from Proposition 2.4.

Corollary 2.6. (Chase, Harrison and Rosenberg) Let $A$ be a commutative ring. $A$ is a Galois extension of $I$ relative to $G$ if and only if the conditions CHR I. and CHR II. hold. 
Proof. Setting $\Gamma=R$ in Theorem 2.5 we have this corollary.

3. Galois theory. In this section, we shall improve Theorem 5 in [4] and develope the Galois theory of separable algebra over a commutafive ring having the indecomposable center by using the Galois theory of commutative indecomposable ring in [3].

Proposition 3.1. Let $\Gamma \subset A$ be algebras over a commutative ring $R, A a$ Galois extension of $\Gamma$ ralative to $G$, and $\Gamma$ a separable algebra over $R$. Then, for every subgroup $H$ of $G$, the $H$-fixed subring $\Lambda^{H}$ of $A$ is also a separable algebra over $R$.

Proof. Since $\Gamma$ is separable over $R$, by Proposition 4 in $[4] \Delta(\Lambda, G)$ is also separable over $R$. For the decompositions of $G$ with respect to $H ; G=H \sigma_{1}+$ $H_{\sigma_{2}}+\cdots+H_{\sigma_{r}}=\sigma_{1}^{\prime} H+\sigma^{\prime} H+\cdots+\sigma_{r}^{\prime} H, \sigma_{1}=\sigma_{1}^{\prime}=1$, we have $\Delta(\Lambda, G)=\sum_{\sigma \in G}$ $\oplus \Lambda \boldsymbol{u}_{\sigma}=\Delta(\Lambda, H) \oplus \sum_{i=2}^{r} \Delta(\Lambda, H) \boldsymbol{u}_{\sigma_{i}}$ and $\sum_{i=2}^{r} \Delta(\Lambda, H) \boldsymbol{u}_{\sigma_{i}}=\sum_{i=2}^{r} \boldsymbol{u}_{\sigma_{i}}^{\prime} \Delta(\Lambda, H)$. We shall show that $\Delta(\Lambda, H)$ is an $R$-separable subalgebra of $\Delta(\Lambda, G)$. Since $\Delta(\Lambda, G)$ is separble over $R, \Delta(A, G)$ is a $\Delta(\Lambda, G)^{e}$-projective module. Now $\Delta(\Lambda, G)^{e}=$ $\Delta(\Lambda, G) \otimes_{R} \Delta(\Lambda, G)^{0}=\sum_{i, j} \oplus \Delta(\Lambda, H)^{e} u_{\sigma_{i}} \otimes u_{\sigma^{\prime}}^{0}$, therefore $\Delta(\Lambda, G)$ is a $\Delta(\Lambda, H)^{e}$-projective module. Since $\Delta(A, H)$ is a direct summand of $\Delta(\Lambda, G)$ as $\Delta(\Lambda, H)^{e}$ -module, $\Delta(A, H)$ is $\Delta(\Lambda, H)^{e}$-projective, therefore $\Delta(\Lambda, H)$ is separable over $R$. On the other hand, $\Lambda$ is a finitely generated projective $\Gamma$-module, hence by Corollary 1 in [4] $\Lambda$ is a finitely generated projective $\Delta(\Lambda, G)$-module. Since $\Delta(\Lambda, G)$ is a $\Delta(\Lambda, H)$-free module, $\Lambda$ is a finitely generated projective $\Delta(\Lambda, H)$ -module. Therefore by Corollary 1 in [4] $\operatorname{Hom}_{\Delta(\Lambda, H)}(\Lambda, \Lambda)=\Delta^{H}$ is separable over $R$.

Using Theorem 2.3 in [3] and Theorem 5 in [4], we have

TheOREM 3.2. Let $A$ and $\Gamma$ be separable algebras over a commutative ring $R$, and suppose that the following conditions are satisfied:

1) The center $C$ of $A$ is indecomposable.

2) There is a finite group $G$ of ring automorphisms of $A$ such that $G$ induces the group of automorphisms of $C$ isomorphic to $G$.

3) $\Gamma$ is the G-fixed subring of $\Lambda$.

4) $\Lambda$ is finitely generated and projective over $R$.

Then $A$ is a Galois extension of $\Gamma$ relative to $G$, and there is a $1-1$ dual cor- 
respondence between subgroups of $G$ and $R$-separable subalgebra of $A$ containing $\Gamma$ in the usual sense of Galois theory.

Proof. Simce $\Lambda$ is separable and finitely generated projective over $R$, the center $C$ of $\Lambda$ is finitely generated projective and separable over $R$. From Theorem 2.3 in [3], the indecomposable ring $C$ is a Galois extension of the $G$-fixed subring $S$ of $C$ relative to $G$. From Theorem 5 in [4], $\Lambda$ is a Galois extension of $T$ relative to $G$. By Proposition 3.1, for every subgroup $H$ of $G$ the $H$-fixed subring $\Lambda^{H}$ is separable over $R$, and $\Lambda$ is a Galois extension of $\Lambda^{H}$ relative to $H$ by Theorem 5 in [4]. Conversely, for every separable subalgebra $\Omega$ over $R$ such that $\Gamma \subset \Omega \subset \Lambda, \Omega$ is separable over $S$, and Proposition 6 in [4] holds for the indecomposable ring $C$ by Theorem 3.3 in [3], hence by the same argument as in Theorem 5 in [4] we have that $A$ is a Galois extension of $\Omega$ relative to a subgroup of $G$. Therefore $\Omega$ is the fixed subring of $\Lambda$ by a subgroup of $G$. Thus we obtain a Galois theory for separable algebra over $R$.

4. Automorphisms of Galois extension. In this section, we assume that $\Lambda$ is a central separable algebra over $C, G$ is a finite group of ring automorphisms of $A$ which induces the group of automorphisms of $C$ isomorphic to $G$, and for the $G$-fixed subring $R$ of $C, C$ is a Galois extension of $R$ relative to $G$. Then by Theorem 5 in [4] $\Lambda$ is a Galois extension of $\Gamma$ relative to $G$, where $\Gamma$ is the $G$-fixed subring of $A$.

Lemma 4.1. Let $C$ be a ring, $M$ a projective $C$-module. For any subset $x_{1}$, $x_{2}, \ldots x_{n}$ in $M$, in which at least one element $x_{i}$ is not zero, there exist elements $c_{1}, c_{2}, \ldots c_{n}$ in $C$ such that at least one of $c_{i}{ }^{\prime} s$ is not zero and $\sum_{i=1}^{n} x_{i} y_{j}=0$ with $y_{i} \in C$ implies always $\sum_{i=1}^{n} c_{i} y_{i}=0$.

Proof. we can prove the lemma similarly to Lemma 6 in [4].

PROPOSITION 4.2. Let $\rho$ be a ring automorphism of $\Lambda$ which leaves invariant each element of $\Gamma$. Then we have $\rho=\sum_{\sigma=\sigma} \lambda_{s} \sigma$ where $\left\{\lambda_{\sigma}\right\}$ is a family of orthogonal idempotents in the center $C$, and $1=\sum_{\sigma \in G} \lambda_{\sigma}$. Furthermore, if $\sigma \neq \tau$ and $\lambda_{\sigma} \neq 0 \lambda_{\tau} \neq$ 0 , then $\sigma^{-1}\left(\lambda_{\sigma}\right) \neq \tau^{-1}\left(\lambda_{\tau}\right)$.

Proof. Since $\rho \in \operatorname{Hom}_{\Gamma}^{r}(\Lambda, \Lambda) \cong \Delta(\Lambda, G)=\sum_{\sigma \in G} \oplus A u_{0}$, we have $\rho=\sum_{\sigma \in G} \lambda_{s} \sigma$ with $\lambda_{\sigma} \in \Lambda$. For any $x$ in $\Lambda, \rho \cdot x=\rho(x) \cdot \rho$, therefore $\sum_{\sigma \in G} \lambda_{\sigma} \cdot \sigma(x) \cdot \sigma=\left(\sum_{\tau \in G} \lambda_{\tau} \tau(x)\right) \cdot$ 
$\left(\sum_{\sigma \in G} \lambda_{\sigma} \sigma\right)$, and we obtain

(*) $\quad \lambda_{\sigma} \sigma(x)=\sum_{\tau \in G} \lambda_{\tau} \tau(x) \lambda_{\sigma} \quad$ for $x \in \Lambda$ and $\sigma \in G$.

If $x$ is taken in $C$, then $\lambda_{\sigma} \sigma(x)=\sum_{\tau \in G} \lambda_{\tau} \lambda_{\sigma} \tau(x)$, therefore $\sum_{\substack{\sigma \neq \tau \tau \\ \tau \in G}} \lambda_{\tau} \lambda_{\sigma} \tau(x)+\left(\lambda_{\sigma}^{2}-\lambda_{\sigma}\right) \sigma(x)$ $=0$ for any $x$ in $C$. By Lemma 4.1 and linearly independence of $\{\sigma\}_{\sigma \in G}$ over $C$, we obtain $\lambda_{\sigma}=\lambda_{\sigma}^{2}$ and $\lambda_{\tau} \lambda_{\sigma}=0$ for $\tau \neq \sigma$. Therefore $\left\{\lambda_{\sigma}\right\}$ is a family of orthogonal idempotents and $\sum_{\sigma \in G} \lambda_{\sigma}=1$ since $\rho(1)=1$. On the orther hand, from $(*)$ we have for $x, y$ in $\Lambda, \lambda_{\sigma} \sigma(x y)=\sum_{\tau \in G} \lambda_{\tau} \tau(x y) \lambda_{s}$, and we have $\lambda_{\sigma} \cdot \sigma(x) \cdot \sigma(y)=\sum_{\tau \in G}$ $\lambda_{\tau} \tau(x) \lambda_{\sigma} \tau(y)$ for any $x \in \Lambda$ and $y \in C$. By the same reason as above, we have $\lambda_{\sigma} \cdot \sigma(x)=\lambda_{\sigma} \sigma(x) \lambda_{\sigma}$ and $\lambda_{\tau} \cdot \tau(x) \cdot \lambda_{\sigma}=0$ for $\tau \neq \sigma$, therefore $\lambda_{\sigma} x=\lambda_{\sigma} \cdot x \lambda_{\sigma}$ for every $x$ in $\Lambda$. On the other hand, for any $x$ in $\Lambda, x \lambda_{\sigma}=\sum_{\tau \in G} \lambda_{\tau} x \lambda_{\sigma}=\sum_{\tau \in G} \lambda_{\tau} x \lambda_{\tau} \lambda_{\sigma}=\lambda_{\sigma} x \lambda_{\sigma}$, therefore $\lambda_{\sigma}$ is contained in the center $C$ of $\Lambda$. Since $\rho(\Lambda)=\Lambda$, for any $y$ in $\Lambda$ there exist $x$ in $\Lambda$ such that $y=\rho(x)=\sum_{\sigma \in G} \lambda_{\sigma} \cdot \sigma(x)$. Since $\lambda_{\sigma} y=\lambda_{\sigma} \cdot \sigma(x)=\sigma\left(\sigma^{-1}\left(\lambda_{\sigma}\right) \cdot x\right)$ for each $\sigma$ in $G$, it follows that $\sigma^{-1}\left(\lambda_{\sigma} y\right)=\sigma^{-1}\left(\lambda_{\sigma}\right) \cdot x$ for each $\sigma \in G$. Accordingly, if $\lambda_{\sigma}$ and $\lambda_{\tau}$ are non zero and $\sigma \neq \tau$, then $\sigma^{-1}\left(\lambda_{\tau}\right) \neq \tau^{-1}\left(\lambda_{\tau}\right)$. Because, if $\sigma^{-1}\left(\lambda_{\sigma}\right)$ $=\tau^{-1}\left(\lambda_{\tau}\right)$ then $\sigma^{-1}\left(\lambda_{r} y\right)=\tau^{-1}\left(\lambda_{\tau} y\right)$. Put $y=\lambda_{\sigma}$, and we have $\sigma^{-1}\left(\lambda_{s}\right)=\sigma^{-1}\left(\lambda_{\sigma}^{2}\right)=$ $\tau^{-1}\left(\lambda_{\tau} \lambda_{\sigma}\right)=\tau^{-1}(0)=0$, it is a contradiction.

Corollary 4.3. If the center $C$ of $A$ is indecomposable, then any $\Gamma$-ring automorphism of $A$ is contained in $G$.

Proposition 4.4. If there are orthogonal indecomposable idempotent elements $e_{1}, e_{2} \ldots e_{n}$ in $C$ such that $\sum_{i=1}^{n} e_{i}=1$, and if there exist $\sigma_{1}, \sigma_{2}, \ldots \sigma_{n}$ of $G$ such that $\sigma_{i}^{-1}\left(e_{i}\right) \neq \sigma_{j}^{-1}\left(e_{j}\right)$ for $i \neq j$, then $\rho=\sum_{i=1}^{n} e_{i} \sigma_{i}$ is a $\Gamma$-ring automorphism of $\Lambda$.

Proof. $\rho$ is clearly a ring endomorphism, and leaves invariant each element of $\Gamma$. Now, we shall show that $\rho$ is an epimorphism of $A$ to $A$. Since $e_{i}$ is indecomposable in $C$ and $\sum_{i=1}^{n} e_{i}=1, \sigma_{j}^{-1}\left(e_{i}\right)$ is also indecomposable in $C$ and $\sum_{j=1}^{n} \sigma_{i}^{-1}\left(e_{j}\right)=1$ for each $i$, therefore $\sigma_{i}^{-1}\left(e_{i}\right)$ is one of $\left\{e_{k}\right\}$. But, $\sigma_{i}^{-1}\left(e_{i}\right) \neq \sigma_{j}^{-1}\left(e_{j}\right)$ for $i \neq j$, hence $\left\{e_{1}, e_{2}, \ldots e_{n}\right\}=\left\{\sigma_{1}^{-1}\left(e_{1}\right), \sigma_{2}^{-1}\left(e_{2}\right), \ldots \sigma_{n}^{-1}\left(e_{n}\right)\right\}$. Therefore $1=$ $\sum_{i=1}^{n} \sigma_{i}^{-1}\left(e_{i}\right)$, and $\sigma_{i}^{-1}\left(e_{i}\right) \cdot \sigma_{j}^{-1}\left(e_{j}\right)=0$ for $i \neq j$. For any $y$ in $A$, put $x_{i}=\sigma_{i}^{-1}\left(e_{i}\right) \cdot \sigma_{i}^{-1}(y)$, $i=1,2, \ldots n$, and $x=\sum_{i=1}^{n} x_{i}$, then $\sigma_{j}^{-1}\left(e_{j}\right) x_{i}=0$ for $i \neq j$, and $\sigma_{i}^{-1}\left(e_{i}\right) x_{i}=x_{i}$. Hence $\rho(x)=\sum_{i=1}^{n} e_{i} \sigma_{i}(x)=\sum \sigma_{i}\left(\sigma_{i}^{-1}\left(e_{i}\right) x\right)=\sum_{i} \sigma_{i}\left(x_{i}\right)=\sum_{i} \sigma_{i}\left(\sigma_{i}^{-1}\left(e_{i}\right) \sigma_{i}^{-1}(y)\right)=\sum_{i=1}^{n} e_{i} y=y . \quad$ Thus 
$\rho$ is an epimorphism.

We shall prove that $\rho$ is a monomorphism. The following proof of this part is due to Professor H. Nagao. If $\rho(x)=0$, then $\sigma_{j}^{-1}(\rho(x))=\sum_{i \neq j} \sigma_{j}^{-1}\left(e_{i}\right)_{\sigma_{j}^{-1}}\left(\sigma_{i}(x)\right)$ $+\sigma_{j}^{-1}\left(e_{j}\right) x=0$ for each $j$, and $\sigma_{j}^{-1}\left(e_{j}\right) \sigma_{j}^{-1}(\rho(x))=\sigma_{j}^{-1}\left(e_{j}\right) x=0$ for $j=1,2, \ldots n$, therefore $x=\sum_{j=1}^{n} \sigma_{j}^{-1}\left(e_{j}\right) x=0$. Accordingly, $\rho$ is an automorphism of $A$.

\section{ReFerences}

[1] M. Auslander and O. Goldman. The Brauer group of a commutative rings, Trans. Amer. Math. Soc. Vol. 97 (1960), 367-409.

[2] - - Maximal order, Trans. Amer. Math. Soc. Vol. 97 (1960), 1-24.

[ 3 ] S. U. Chase, D. K. Harrison and A. Rosenberg, Galois theory and Galois cohomology of commutative rings. Memoirs Amer. Math. Soc. No. 52 (1965).

[4] T. Kanzaki, On commutor ring and Galois theory of separable algebras, Osaka J. Math. Vol. 1 (1964), 103-115.

\section{Osaka Gakugei Daigaku}

